\title{
A Novel Algorithm to Estimate the Reliability of Hybrid Computer Communication Networks
}

\author{
Yasir Khalil Ibrahim \\ Department of Communications and Electronics Engineering, College of Engineering, Jerash University, Jerash, Jordan. \\ Email: dr.yasir.khalil@gmail.com
}

Received August 21 ${ }^{\text {st }}$, 2013; revised September 20 ${ }^{\text {th }}$, 2013; accepted September $30^{\text {th }}$, 2013

Copyright (c) 2013 Yasir Khalil Ibrahim. This is an open access article distributed under the Creative Commons Attribution License, which permits unrestricted use, distribution, and reproduction in any medium, provided the original work is properly cited.

\begin{abstract}
In this paper, we proposed an algorithm to estimate the reliability of a hybrid computer communication network. A hybrid computer network is a network that consists of wire and wireless sub networks. The proposed algorithm is built upon using a simulation model due to the complexity of the network topology. We tested the proposed algorithm, and the results show consistency in the reliability estimates.
\end{abstract}

Keywords: Reliability; Hybrid Networks; Wireless Subnetworks; Wier Sub Networks

\section{Introduction}

The recent advances in building computer communications networks encourage researchers in the field to model the performance of the existing networks, and the advantage behind performance modeling is to provide the specialists in industry with models and tools that can help them to test computer communication networks during design stage before installation stage, and test the networks under different conditions. The main advantage of testing the proposed networks under different conditions is to lower failure rates, specifically if the networks under consideration are serving different sectors. In terms of modeling, the open question is: what is a hybrid network?

The hybrid network consists of a set of computers and a set of links (see Figure 1), where those computers are connected based on a well defined topology. The links are either wire or wireless links. In this context, the word hybrid is defined in terms of link types. Thus, the network under consideration consists of wire sub networks and wireless sub networks.

In terms of modeling, the hybrid network is defined as a undirected graph $G(t)=(V(t) ; E(t))$ that consists of a set of nodes $V(t)=f v_{1}^{(t)}, v_{2}^{(t)}, \cdots, v_{n}^{(t)} g$ and a set of edges (i.e. links) $E(t)=f e_{1}^{(t)}, e_{2}^{(t)}, \cdots, e_{k}^{(t)} g$, where the computers are connected based on a well defined topology, and the topology is varying over the time $t$. The reliability of computer communication network is defined in terms of probability. The reliability of computer communication network is defined as the probability that the network is connected during a specific period of time $\Delta t$. Let $P_{r}$ be a probability measure, the network reliability is defined as: $P_{r}$ (the network is connected during $\Delta t)$.

To estimate the required reliability, we have to monitor the network conditions at specific points in time $\Delta t$, and conclude the network performance based on those evaluations. To explain this concept, we present the following example. Consider a hybrid network that consists of 20 nodes and 6 wire links and 13 wireless links, where

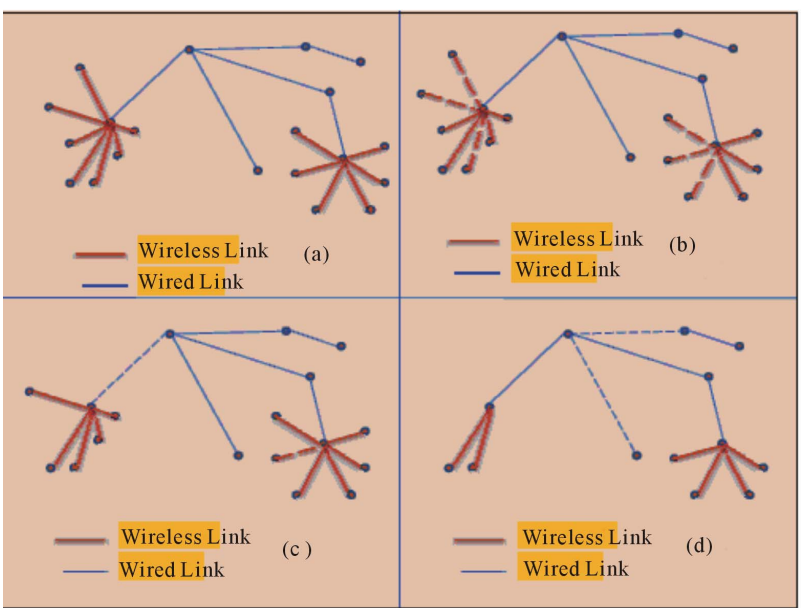

Figure 1. A hybrid computer communication network. 
the disconnected links are represented by dashed lines. The topology is presented in Figure 1. From Figure 1, the network consists of one wire sub network and two wireless sub networks. The Figure 1(a) represents a proposed hybrid computer communication network at time $t_{0}$. As we mentioned, the network condition is varying over the time, and Figures 1((b)-(d)) represent different network conditions at different specific points in time (say at $t_{1}, t_{2}$, and $t_{3}$ respectively).

The open question that arises in this context is: In which way we can estimate the required reliability under the following constrains: 1) the network topology is assumed to be a complicated topology, and 2) the numbers of links and nodes are varying over the time. To answer the question, we have to setup a number of assumptions. Those assumptions are generic assumptions, which are listed below.

1) The node reliability is defined as the probability that the node is operational.

2) The link reliability is defined as the probability that the link is operational.

3) The reliabilities of nodes are assumed to be stochastically independent.

4) The reliabilities of links are assumed to be stochastically independent.

5) Each link has two status: either up or down.

6) Each node has two status: either up or down.

7) At any instant of time, we assume that no repairs occurred for link failure or node failure.

8) No hardware redundancy.

In this section, we just presented the introduction to the problem under consideration. The structure of this paper can be summarized as follows. In Section 2, we present the statement of the problem. In Section 3, we present the related work. The proposed algorithm is presented in Section 4. The experimentations and results are presented in Section 5. finally conclusions are presented in Section 4.

\section{The Statement of the Problem}

The problem under study is a well known problem in the area of computer communication networks, which is the problem of estimating the reliability of computer communication network. The process of building any system can be divided into a number of stages, and one of those stages is the testing stage. Testing a computer communication network requires software tools. The software tools usually are building using different types of performance models. In this paper, the network under consideration is a hybrid network, and the network topology is assumed to be a complicated topology.

In this section, we just presented the statement of the problem under consideration; in the next section we present the related work.

\section{Related Work}

In this section, we present the related work. In recent years, the network technology is considered as a growing cutting-edge technology, and the degree of reliability and availability of computer communication network has a direct impact on the performance of computer systems that uses the network as an environment. The models used to estimate the reliability of computer communication network can be classified as either: 1) theoretical models or 2) empirical models. The system reliability [1] is defined as the probability that the system (e.g. network) is operational without failures during a specific period of time. The network reliability is defined as the probability that the nodes can establish successful communications during a specific period of time [2,3]. Precisely, Let $V$ be a set of operational nodes and let $E$ be a set of operational links, and let $\Omega$ be the set of operational states, then the network reliability is defined as:

$$
R^{\prime}=\sum_{\Omega}\left[\Pi_{e_{i} \in e} p_{i}\right]\left[\Pi_{e_{i} \in e^{c}}\left(1-p_{i}\right)\right]\left[\Pi_{v j \in v} q_{j}\right]
$$

where $q_{j}$ is the reliability of node $j$ and $p_{i}$ is the reliability of link $i$. As the complexity of the network topology increases, it becomes hard to compute the network reliability using the theoretical models. There are other approaches used to estimate the network reliability, and one of those approaches is neural networks. A neural network-based approach proposed by Srivaree-ratana et al. [4] to estimate the network reliability.

A simulation-based models have been used to estimated the reliability of different systems (e.g. mobile agents based systems, distributed systems), where the environment of those systems is the computer communication network. For example, Mosaab Daoud and Qusay Mahmoud [3,5] estimated the dependable performance of the mobile agents-based system using a simulation model.

Takeshi Koide [6] and others proposed an algorithm to compute marginal reliability importance for network systems with k-terminal reliability efficiently. Marginal reliability importance is an appropriate quantitative measure on a system component against system reliability and it contributes to design of reliable systems. Computing marginal reliability importance in network systems is time-consuming due to its NP-hardness. Zuo and others [7] in multistate networks, evaluating the probability, in such networks, that the flow from the source node to the sink node is equal to or greater than a demanded flow of $d$ units. A general method for reliability evaluation of such multistate networks is using minimal path (cut) vectors. Al Khateeb and S. Al-Irhayim [8], proposed a reliability enhancement of complex networks through redundancy scaling.

In this section, we presented the related work, in the 
next section, we present the proposed algorithm.

\section{The Proposed Algorithm}

The network conditions are varying over the time. In fact, the network status is defined as a mapping into the range $(0,1)$, which means that either the network is disconnected or connected. The connectivity is classified as either fully connected network or partially connected network. The fully connected network is a network where each node that belongs to the network can send information to every node that belongs to the same network. In other words, there exists a path between any pair of nodes through which the communications between those pair of nodes can be established. The partially connected network is a network where a subset of nodes can only send information (i.e. connected) via the network, which implicitly the network status can be classified as: the network is partially operational.

As we mentioned earlier in this section, the network consists of a set of nodes and a set of links. A node is either up or down, and a link is either up or down. The reliability of the network is defined in terms of the full connectivity of the network during a specific period of time. Thus, the estimation of the reliability can be achieved by building a simulation model, through which we can estimate the required reliability. Specifically, when the network topology is a complicated topology, and it is varying over the time, this situation makes the theoretical models hard to be implemented. To propose the required model, we have to define a set of variables, where each variable represent the status of a component (i.e. node, link, sub network, entire network). In the next part of this section, we present the basic definitions.

Definition 1: Given a hybrid computer communication network $G(t)=(V(t) ; E(t))$, where its topology is varying over the time $t$. Let $V(t)$ be a set of nodes at time $t$. Let $E(t)$ be a set of links at time $t$. Let $X_{i}(t)$ be a binary variable which represents the status of node $i$ at time $t$, where $i=1, \cdots, j V(t) j$. The node status is either up or down. Let $Y_{j}(t)$ be a binary variable which represents the status of link $j$ at time $t$, where $i=1, \cdots, j E(t) j$. The link status is either up or down. Let Status ( $t$ ) be a binary variable which represents the status of the hybrid computer communication network at time $t$, where $t=t_{1}, \cdots, t_{r}$. The network status is either connected or disconnected. The variable Status $(t)$ at a time $t$ is defined in terms of the variables $X_{i}(t)$ and $Y_{j}(t) 8 i, 8 j$.

Definition 2: Given a hybrid computer communication network $G(t)=(V(t) ; E(t))$, where its topology is varying over the time $t$. The reliability of

$$
G^{\text {subgraph }}(t)=\left(V^{\prime}(t) ; E(t)\right),
$$

where $V^{\prime}(t)$ b $V(t)$, is called the partial reliability of
$G(t)=(V(t) ; E(t))$.

To proceed further in solving the problem under consideration, we build a simulation model (see Algorithm 1) to estimate the required reliability. The model uses random numbers to simulate the status of the network conditions. The proposed model has the following parts:

1) The node conditions are generated using random numbers, where each node is defined by two status: either up or down.

Algorithm 1: Estimating the reliability of a hybrid computer communication network.

Input: Given undirected graph that represents the hybrid computer communication network. $G(t)=(V(t) ; E(t))$ associated with the set of links reliabilities $P$ and the set of nodes reliabilities $Q$.

Output: The estimated reliability of the hybrid computer communication network $R^{\Lambda}$.

Let $X_{i}(t)$ be a binary variable which represents the status of node $i$ at time $t$, where $i=1, \cdots,|V(t)|$. The node status is either up or down.

Let $Y_{j}(t)$ be a binary variable which represents the status of link $j$ at time $t$, where $i=1, \cdots,|E(t)|$. The link status is either up or down.

Let Status $(t)$ be a binary variable which represents the status of the hybrid computer communication network at time $t$, where $t=t_{1}, \cdots, t_{r}$. The network status is either connected or disconnected.

For $t=t_{1}$ to $t_{r}$ do, Generate the status of the network at time $t$; for $i=1$ to $|V(t)|$ do, Generate a random number between 0 and 1 (i.e. rnd $\in[0,1]$ ). If rnd $\in\left[0, p_{i}\right]$, where $p_{i} \in P$, then set $X_{i}(t)=1$, otherwise $X_{i}(t)=0$; for $j=1$ to $|E(t)|$ do, Generate a random number between 0 and 1 (i.e. rnd $\in[0,1]$ ). If rnd $\in\left[0, q_{i}\right]$, where $q_{i} \in Q$, then set $Y_{j}=1$, otherwise $Y_{j}=0$, end.

Let

$$
V^{\prime}(t)=\left\{v_{i} \mid v_{i} \in V(t) \text { such that } X_{i}(t)=1\right\} ;
$$

Let

$$
E^{\prime}(t)=\left\{e_{j} \mid e_{j} \in E(t) \text { such that } Y_{j}(t)=1\right\} ;
$$

Set Status $(t)=1$ if $G^{\prime}(t)=V^{\prime}(t), E^{\prime}(t)$ is fully connected, Otherwise set Status $(t)=0$,

$$
\begin{gathered}
T=\sum_{t=1}^{r} \operatorname{status}(t), \\
\dot{R}=\frac{T}{r},
\end{gathered}
$$

end.

2) The link conditions are generated using random numbers, where each link is defined by two status: either up or down.

3) The network conditions, which are defined by the network connectivity. The network connectivity can be tested by using one of the existing graph theory algorithms. 
4) The estimated reliability is defined as the long-term average of number of times where the network is connected to the total number of times the network connectivity is tested (i.e. number of time the network is connected add it to number of times the network is disconnected).

In this section, we presented the basic definitions and the algorithm required to estimate the reliability of hybrid computer communication network. We built the algorithm using a simulation model instead of the theoretical models. The main reason behind using simulation model is the complexity of the network topology. In the next section, we present experimentation and results.

\section{Experimentation and Results}

In this section, we present the experiments performed to estimate the required reliability. The computer communication network under consideration is a hybrid network (i.e. wireless and wire links are considered). The wireless network has the bipartite topology, where the nodes are divided into two sets, one set has the cardinality one and the other set has the cardinality $([V(t) 1])$. The set of a singleton node represents the wireless access point, which connected with the wire sub network (i.e. the sub network with fixed wire topology). The recent hardware specifications of the existing wireless access points indicated that the average number of computers (i.e. the second set of nodes) is approximately 50 computers. The topology of the wire sub network is illustrated in Figure 2. The topology of the wireless sub network is illustrated in Figure 3.

To estimate the required reliabilities, we performed two experiments. Using the law of large number, we estimated the required reliability based on 1000 iterations, and we computed the reliability based on the average of 40 values (i.e. estimates). The results are given in Tables 1 and 2 respectively. In the first experiment, we assigned

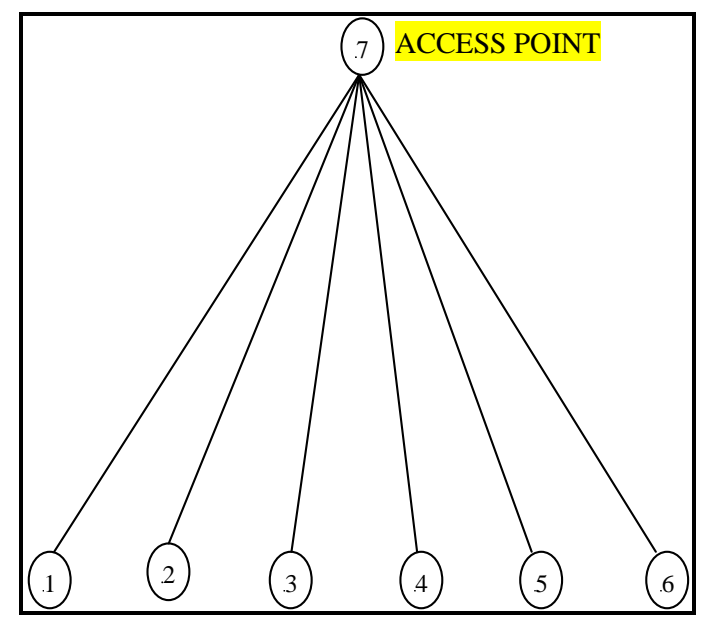

Figure 2. Wireless sub network.

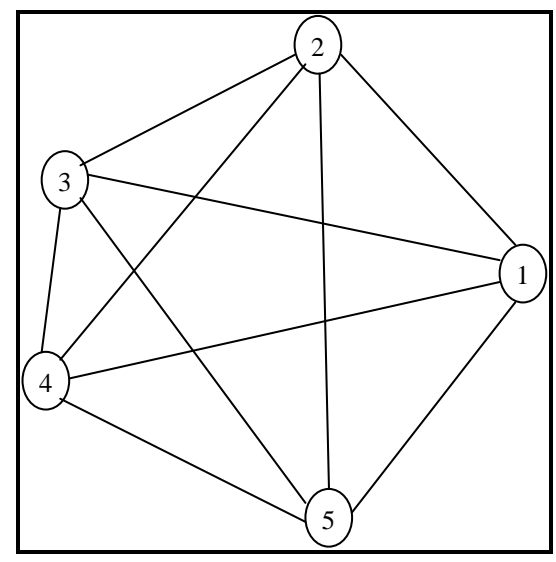

Figure 3. Wire sub network.

the values $0.9,0.92,0.94,0.96$, and 0.98 to the reliability of wireless links, and we assigned the values $0.6,0.62$, $0.64,0.66$, and 0.68 to reliability of wire links (see columns 1, 2 Table 1). We estimated the reliability of the wireless subnetwork using the proposed algorithm (Algorithm 1). The results are listed in Table 1-column 3. In addition, we estimated the reliability of the wireless sub network using the proposed algorithm (Algorithm 1). The results are listed in Table 1-column 4. Finally, by assuming that the hybrid network consists of one wireless sub network and one wire sub network, the estimated reliabilities of the hybrid network are listed in Table 1-column 5. Based on the standard deviation of 40 estimates of the network reliability (see Table 1-columns 6, 7), the proposed algorithm shows consistency in estimating the required reliability.

In the second experiment, we estimated the reliability of the same hybrid computer communication network, and we assigned different reliabilities to the wire links, where those values are: 4.0, 4.2, 4.4, 4.6, and 4.8. The estimated reliabilities are given in Table 2 . Based on the standard deviation of 40 estimates of the network reliability (see Table 2-columns 6, 7), the proposed algorithm shows consistency in estimating the required reliability.

To proceed further in testing the proposed algorithm, we considered another factor, which is the network topology. The network topology is expected to be a significant factor in estimating the required reliability. Without loss of generality, assume that we have a hybrid computer communication network, which is consists of two subnetworks:

One wireless subnetwork and one wire subnetwork. Suppose that the wireless network is the subnetwork illustrated in Figure 2. Assume that the topology of the wire network is given in Figure 4, which is different from the topology given in Figure 3. The number of nodes of the wire subnetwork given in Figure 2 is less then the number of nodes of the wire subnetwork given 
in Figure 4. In addition, the number of links of the wire subnetwork given in Figure $\mathbf{2}$ is greater than the number of links of the wire subnetwork given in Figure 4. The reliabilities of nodes are assigned the value one. The reliabilities of the wire links are the same for both wire subnetworks (see Tables 1 and 3-column 2). Therefore, the wire subnetwork with higher number of links is more reliable than the wire subnetwork with fewer links (see Tables 1 and 3-column 4). Hence, the topology of the network has a significant impact on the network reliability.

In this section, we presented the experimentations and results. In the next section the conclusions are presented.

\section{Conclusion}

One of the interesting problems in the field of computer communication networks is the problem of estimating the network reliability. In this paper, we proposed a robust algorithm for estimating the reliability of hybrid com-

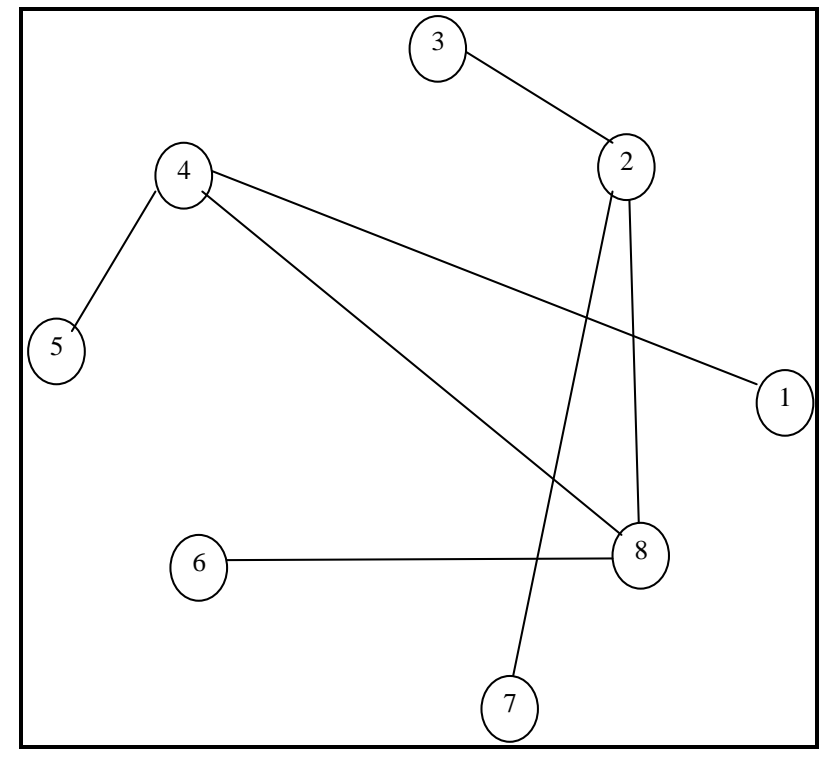

Figure 4. Wire subnetwork.

Table 1. The estimated reliability of a hybrid computer communication network.

\begin{tabular}{ccccccccc}
\hline Link reliability (wireless) & Link reliability (wire) & $\begin{array}{c}\text { Estimated reliability for } \\
\text { wireless sub network }\end{array}$ & $\begin{array}{c}\text { Estimated reliability for } \\
\text { wire sub network }\end{array}$ & $R^{\wedge}$ & $\begin{array}{l}\sigma \\
R^{\wedge}\end{array}$ wireless $\begin{array}{l}\sigma \\
R^{\wedge} \text { wired }\end{array}$ \\
\hline 0.9 & 0.6 & 0.0057 & 0.9190 & 0.0052 & 0.0022 & 0.0085 \\
0.92 & 0.62 & 0.0153 & 0.9344 & 0.0143 & 0.0043 & 0.0085 \\
0.94 & 0.64 & 0.0457 & 0.9465 & 0.0433 & 0.0046 & 0.0062 \\
0.96 & 0.66 & 0.1313 & 0.9562 & 0.1255 & 0.0096 & 0.0066 \\
0.98 & 0.68 & 0.3616 & 0.9664 & 0.3495 & 0.0134 & 0.0049 \\
\hline
\end{tabular}

Table 2. The estimated reliability of a hybrid computer communication network.

\begin{tabular}{cccccccc}
\hline $\begin{array}{c}\text { Link reliability } \\
\text { (wireless) }\end{array}$ & $\begin{array}{c}\text { Link reliability } \\
\text { (wire) }\end{array}$ & $\begin{array}{c}\text { Estimated reliability } \\
\text { for wireless sub network }\end{array}$ & $\begin{array}{c}\text { Estimated reliability } \\
\text { for wire sub network }\end{array}$ & $R^{\wedge}$ & $\begin{array}{l}\sigma \\
R^{\wedge}\end{array}$ & wireless & $\begin{array}{l}\sigma \\
R^{\wedge} \text { wired }\end{array}$ \\
\hline 0.9 & 0.4 & 0.0057 & 0.6334 & 0.0036 & 0.0022 & 0.0152 \\
0.92 & 0.42 & 0.0153 & 0.6758 & 0.0103 & 0.0043 & 0.0148 \\
0.94 & 0.44 & 0.0457 & 0.7131 & 0.0326 & 0.0046 & 0.0114 \\
0.96 & 0.46 & 0.1313 & 0.7437 & 0.0976 & 0.0096 & 0.0141 \\
0.98 & 0.48 & 0.3616 & 0.7790 & 0.2817 & 0.0134 & 0.0125 \\
\hline
\end{tabular}

Table 3. The estimated reliability of a hybrid computer communication network.

\begin{tabular}{ccccccc}
\hline $\begin{array}{c}\text { Link reliability } \\
\text { (wireless) }\end{array}$ & $\begin{array}{c}\text { Link reliability } \\
\text { (wired) }\end{array}$ & $\begin{array}{c}\text { Estimated reliability for } \\
\text { wireless subnetwork }\end{array}$ & $\begin{array}{c}\text { Estimated reliability for } \\
\text { wire subnetwork }\end{array}$ & $\dot{R}$ & $\sigma \dot{R}_{\text {wirless }}$ & $\sigma \dot{R}_{\text {wire }}$ \\
\hline 0.9 & 0.6 & 0.0057 & 0.0466 & $2.6562 \mathrm{e}-004$ & 0.0022 & 0.0076 \\
0.92 & 0.62 & 0.0153 & 0.0562 & $8.5986 \mathrm{e}-004$ & 0.0043 & 0.0080 \\
0.94 & 0.64 & 0.0457 & 0.0701 & 0.0032 & 0.0046 & 0.0068 \\
0.96 & 0.66 & 0.1313 & 0.0839 & 0.0110 & 0.0096 & 0.0086 \\
0.98 & 0.68 & 0.3616 & 0.0993 & 0.0359 & 0.0134 & 0.0084 \\
\hline
\end{tabular}


puter communication network, where the network consists of wireless sub networks connected to wire sub networks. When the structure complexity (i.e. topology) of the network increases, it is hard to estimate the required reliability by using theoretical models, and instead, we built the algorithm by using a simulation-based model. The proposed algorithm produced consistency estimates. In the future work we are aiming to study the impact of the topology (i.e. structure) of the network on the network reliability.

\section{Acknowledgements}

We performed the experiments using the graph theory tool box Matgraph: A Graph Theory Toolbox for MATLAB, (http://www.ams.jhu.edu/ ers/matgraph/).

\section{REFERENCES}

[1] M. L. Shooman, "Reliability of Computer Systems and Networks: Fault Tolerance, Analysis, and Design,” John Wiley and Sons, Inc., New York, 2002.

[2] F. Altiparkmak, B. Dengiz and A. Smith, "Reliability Optimization of Computer Communication Networks Using Genetic Algorithms," Proceedings of the 1998 IEEE International Conference on Systems, Man and Cybernetics, San Diego, October 1998, pp. 4676-4681.

[3] M. Daoud and Q. Mahmoud, "Monte Carlo Simulation-
Based Algorithms for Estimating the Reliability of Mobile Agent-Based Systems," Journal of the Network and Computer Applications, Vol. 31, No. 1, 2008, pp. 19-31. http://dx.doi.org/10.1016/j.jnca.2006.06.007

[4] C. Srivaree-Ratana, A. Konak and A. Smith, "Estimation of All-Terminal Network Reliability Using an Artificial Neural Network," Computer Operation Research, Vol. 29, No. 7, 2002, pp. 849-868. http://dx.doi.org/10.1016/S0305-0548(00)00088-5

[5] M. Daoud and Q. Mahmoud, "A Fuzzy Approach to Reliability Estimation of Mobile Agent-Based Systems," IEEE International Conference on Systems, Man and Cybernetics, 2007, pp. 2854-2859.

[6] T. Koide, S. Shinmori and H. Ishii, "Efficient Computation of Network Reliability Importance on k-Terminal Reliability,” International Journal of Reliability, Quality and Safety Engineering, Vol. 12, No. 3, 2005, p. 213. http://dx.doi.org/10.1142/S0218539305001793

[7] M. J. Zuo, Z. Tian and H.-Z. Huang, “An Efficient Method for Reliability Evaluation of Multistate Networks Given all Minimal Path Vectors," IIE Transactions (Institute of Industrial Engineers), Vol. 39, No. 8, 2007, pp. 811-817.

[8] W. Al Khateeb and S. Al-Irhayim, "Reliability Enhancement of Complex Networks through Redundancy Scaling," Proceedings of the International Conference on Computer and Communication Engineering, Kuala Lumpur, 2010, pp. 11-13. 